\title{
O Ensino de Habilidades e Atitudes: um Relato de Experiências
}

\author{
Teaching of Attitudes and Skills: an \\ Experience Report
}

\author{
Sandra Odebrecht Vargas Nunes \\ Evelin Massae Ogatta Muraguchi \\ Olavo Franco Ferreira Filho \\ Rose Meire Albuquerque Pontes ${ }^{I}$ \\ Lucienne Tibery Queiroz Cardoso ${ }^{I}$ \\ Cintia Magalhães Carvalho Grion ${ }^{I}$ \\ Renata Maciulis Dip ${ }^{I}$ \\ Luiz Carlos Lúcio Carvalho
}

\author{
PALAVRAS-CHAVE: \\ - Ensino; \\ - Habilidades; \\ - Atitudes; \\ - Aprendizagem Baseada em \\ Problemas; \\ - Avaliação.
}

Recebido em: 13/01/2011

Reencaminhado em: 26/05/2012 Reencaminhado em: 30/08/2012 Reencaminhado em: 06/11/2012 Aprovado em: 03/12/2012

REVISTA BRASILEIRA DE EDUCAÇÃO MÉDICA $126 \frac{117(1): 126-131 ; 2013}{30}$

\section{RESUMO}

Este artigo relata a experiência do ensino de Habilidades e Atitudes, na graduação em Medicina da Universidade Estadual de Londrina (UEL) com a metodologia de ensino da Aprendizagem Baseada em Problemas, ancorada no modelo biopsicossocial. O ensino de Habilidades e Atitudes implica a formulação diagnóstica mutiaxial, descrição contextual e padronizada da condição clínica. Utiliza como instrumento a avaliação sistemática de eixos e domínios altamente informativos e relevantes para o tratamento. Eixo I: transtornos clínicos (mentais e condições médicas gerais); Eixo II: incapacidades nos cuidados pessoais, funcionamento ocupacional e com a família, e funcionamento social mais amplo; Eixo III: fatores contextuais (problemas interpessoais e outros psicossociais e ambientais); Eixo IV: qualidade de vida (refletindo primariamente as percepções do próprio paciente). A competência clínica foi avaliada por meio da discussão de casos clínicos, portfólios reflexivos e pelo Exame Clínico Estruturado por Objetivo (Osce), método que avalia as habilidades clínicas, as habilidades de atitudes e a comunicação dos estudantes de Medicina.

This article describes an experience of teaching Attitudes and Skills in a medical course at the Londrina State University using the methodology of Problem-Based Learning grounded in a biopsychosocial model. The teaching of Attitudes and Skills requires from teachers a multi-axial diagnostic formulation in a contextual and standardized description of the clinical condition through a number of highly informati$v e$, therapeutically significant and systematically assessed axes or domains. The assessment of a patient should lead to multi-axial diagnostic formulation in a systematically assessed axes or domains. Axis I: clinical disorders (mental and general medical conditions); Axis II: disabilities (in personal care, occupational functioning, functioning with family, and broader social functioning); Axis III: contextual factors (interpersonal and other psychosocial and environmental problems); Axis IV: quality of life (primarily reflecting patient's self-perceptions). The assessment of clinical competence was performed through the discussion of clinical cases, the use of reflexive portfolios, and Objective Structured Clinical Exams (Osce), a method to evaluate the medical students' clinical skills, attitudes and communications skills. 


\section{INTRODUÇÃO}

O ensino de Habilidades e Atitudes no curso de Medicina da Universidade Estadual de Londrina (UEL) ocorre por meio da metodologia de ensino da Aprendizagem Baseada em Problemas (ABP ou PBL) e é ancorado no modelo biopsicossocial. Este modelo se caracteriza pela adição do processo cognitivo ao modelo explicativo dedutivo e geralmente se inicia pela observação que desperta emoções, tem uma representação cognitiva e gera uma explicação e compreeensão de causalidade ${ }^{1}$.

O processo de ensinar e avaliar Habilidades e Atitudes no curso de Medicina é complexo e deve ser visto com uma perspectiva biopsicossocial. Os princípios da avaliação das aprendizagens das habilidades clínicas, de atitudes e de comunicação devem ser aplicados de maneira repetida e com a utilização de vários recursos e diferentes métodos, numa variedade de ambientes e contextos, para capturar diferentes aspectos do desempenho. Utilizam o equilíbrio de situações complexas da vida real que exigem raciocínio e julgamento bem estruturados, com avaliações simplificadas focadas em conhecimentos clinicos cotidianos, habilidades de comunicação e atitudes. Os métodos de avaliação devem incluir a observação direta do comportamento, fornecimento de feedback e monitorização contínua ${ }^{2}$

O ensino de Habilidades e Atitudes pode ocorrer em vários cenários, como na Atenção Primária, nos ambulatórios e nas emergências. Em ambientes de emergência, ricos em incertezas, em que as tomadas de decisões são rápidas, muitas vezes há sobrecarga de trabalho do médico e equipe mal treinada, gerando dados clínicos incompletos e interrupções na relação entre médico e paciente. Neste contexto, é necessário auxiliar o desenvolvimento de autocrítica para o processo diagnóstico, buscando a redução de erros evitáveis ${ }^{3}$.

A prática médica exige múltiplas competências, desde o conhecimento médico, a técnica, a cognição, até os aspectos emocionais. É difícil avaliar estas competências. Um método de avaliação do raciocínio clínico em situações de incerteza foi desenvolvido com base na teoria de scripts ${ }^{4}$. Para aquisição do conhecimento científico e o desenvolvimento moral, é necessário o funcionamento cognitivo, que permite utilizar o conhecimento para a solução de problemas da vida real, a integração do conhecimento biomédico e psicológico para formar o raciocínio clínico, a habilidade de comunicação social e o funcionamento afetivo-moral ${ }^{5}$.

Os docentes do Departamento de Clínica Médica da UEL propuseram um treinamento especial para os alunos do terceiro ano de Medicina com a finalidade de melhorar a prática clínica e aprimorar o processo do ensino-aprendizagem e avaliação da disciplina de Habilidades e Atitudes. No treinamento da aquisição de Habilidades e Atitudes utilizou-se a metodologia ABP ou PBL e se trabalhou com o objetivo de resolver problemas em pequenos grupos de até oito alunos e com um professor/tutor, que era o facilitador do grupo. Os casos clínicos foram os pontos de partida. $\mathrm{O}$ aluno realizou a entrevista baseada na comunicação social, avaliou a história clínica, revisou o prontuário, realizou o exame físico, pesquisou, discutiu com os colegas, com o professor/tutor e outros profissionais, confrontou seus dados com as evidências científicas, formulou hipóteses diagnósticas abrangentes para a tomada de decisões. A aprendizagem do raciocínio clínico e tomada de decisões foi complementada nos grupos tutoriais temáticos ${ }^{6,7}$, nas habilidades e atitudes ${ }^{8,9,10}$, nas práticas de interação entre ensino, serviço e comunidade ${ }^{11}$ e na estratégia de integração entre o ciclo básico e o clínico ${ }^{12}$.

As avaliações da aquisição do conhecimento de Habilidades e Atitudes foram realizadas por meio do Exame Clínico Estruturado por Objetivo (Osce), relatórios de casos clínicos e portfólios reflexivos.

\section{O ENSINO DA DISCIPLINA DE HABILIDADES E ATITUDES}

O ensino de Habilidades e Atitudes em um modelo biopsicossocial deve ser visto como a integração da experiência clínica com a capacidade de analisar e aplicar racionalmente a informação científica ao cuidar de pacientes. Norteada pela Medicina Baseada em Evidências, traduz-se pela prática da medicina em um contexto em que a experiência clínica é integrada com a capacidade de analisar criticamente e aplicar de modo racional a informação científica. O objetivo maior é melhorar a qualidade da assistência médica, e as dúvidas que surgem ao se resolverem problemas de pacientes são os principais estímulos para que se procure atualizar os conhecimentos ${ }^{12}$.

A prática médica é primeiramente uma atividade científica. Logo, as decisões clínicas devem ser baseadas exclusivamente na evidência científica. É redutora e não tem em conta a insubstituível necessidade do julgamento clínico em todas as suas vertentes práticas. O ditado clássico de que "a medicina é uma ciência e uma arte" apoia a necessidade da integração da metodologia da Medicina Baseada em Evidências com a metodologia das artes e humanidades, em busca de uma prática médica satisfatória para os doentes e também para os médicos ${ }^{13}$

Além das grandes descobertas na medicina a respeito de processos biológicos, das causas das doenças, do tratamento de doenças, do desenvolvimento de novos instrumentos para o diagnóstico de doenças, os padrões psicológicos devem ser 
incorporados à medicina, bem como combinar novas hipóteses, questionamentos e o desenvolvimento de novos conceitos. Para explicações de doenças, padrões interdisciplinares devem ser incorporados ${ }^{14}$.

O modelo biomédico que forma o paradigma da resolução de problemas é firmado sobre a geração de hipóteses e a interpretação dos dados clínicos para sua verificação, com reconhecimento de padrões e categorização. O processo de solução de problemas clínicos necessita de investigação diagnóstica e requer ação terapêutica ou preventiva por parte do médico $^{15}$. Do modelo biomédico ao modelo biopsicossocial em saúde, há uma volta da reintegração das dimensões psicossociais às práticas em saúde, proporcionando uma visão integral do ser e do adoecer. O modelo biopsicossocial, ao ser incorporado à formação médica, implica treinamento em relacionamento, vínculo e comunicação ${ }^{16}$.

O ensino das habilidades do raciocínio clínico tem por objetivo que os estudantes do terceiro ano do curso se tornem mais aptos à prática médica de: (1) coletar e analisar informações de pacientes; (2) discutir a anamnese, o exame físico e exames complementares; (3) elaborar os planos diagnósticos multiaxial e terapêutico; (4) estimular a conexão da teoria com a prática, realizar confronto com a literatura tomando por base a medicina baseada em evidências científicas; (5) utilizar avaliações das competências práticas por meio de discussão de caso clínico, avaliação de portfólios reflexivos e Osce. As competências necessárias à prática do raciocínio clínico estão sumarizadas no Quadro 1.

O curso proposto se iniciou por meio de discussão de casos clínicos com a finalidade de integração do conhecimento científico com a prática médica, realização de uma formulação diagnóstica abrangente multiaxial e utilização de intervenções preventivas e terapêuticas. A avaliação das competências clínicas dos alunos ocorreu no contexto clínico prático. Cada docente realizou semanalmente atividade de prática clínica, com seis a oito alunos. Para aquisição de competências clínicas, o aluno deveria realizar anamnese, exame clínico, indicação de exames complementares, formulação de diagnósticos abrangentes, englobando transtornos clínicos, incapacidade, fatores contextuais, qualidade de vida, bem como do plano terapêutico e prognóstico. O raciocínio diagnóstico ocorreu na detecção dos componentes do problema clínico, no uso de linha de raciocínio focalizado nas hipóteses diagnósticas multiaxiais, na interpretação correta dos dados em relação aos padrões de doença previamente estabelecidos, na realização de um processo de diagnóstico multiaxial dos transtornos clínicos, nível de incapacidade, fatores contextuais, aplicação de intervenções preventivas e terapêuticas.
QUADRO 1

Competências necessárias à prática do raciocínio clínico e à tomada de decisões

\begin{tabular}{|l|}
\hline * identificar os problemas relevantes do paciente, para realização \\
da anamnese;
\end{tabular}

As habilidades adquiridas por meio do raciocínio clínico e tomada de decisões, além de aprimorarem a qualidade da anamnese, exame físico, indicação de exames complementares, se ampliaram nas formulações diagnósticas abrangentes, que forneceram uma descrição dos transtornos clínicos, incapacidades, fatores contextuais, qualidade de vida, por meio da avaliação sistemática de eixos e domínios altamente informativos e relevantes para o tratamento.

A boa prática médica requer integração entre teoria e prática. A introdução de métodos didáticos que estimulem o autoaprendizado no curso de Medicina da UEL procurou contribuir para formar profissionais capazes de selecionar adequadamente a fonte do conhecimento e transferir a informação para a prática médica. Além do mais, é importante que o ensino da prática médica capacite o estudante a pesquisar e criticar a informação científica, contribuindo para desenvolver atitudes que resultem na melhoria da qualidade dos cuidados que esse aluno venha prestar a seus pacientes ao se tornar médico. 


\section{AVALIAÇÃO DAS HABILIDADES E ATITUDES NO MODELO PEDAGÓGICO DE APRENDIZAGEM BASEADA EM PROBLEMAS}

A avaliação do desempenho do aluno em Habilidades e Atitudes implica avaliar vários domínios de competência clínica, do comportamento, da comunicação com pacientes e familiares, bem como o fornecimento de feedback.

A avaliação do progresso obtido na aquisição do conhecimento das Habilidades e Atitudes se baseia no Osce, na observação de prática clínica, discussão de casos clínicos e avaliação de portfólios reflexivos. Na avaliação, é importante o feedback imediato que deve ser fornecido ao estudante, de modo a configurar o processo de obtenção de dados de seus progressos individuais. O Osce permite avaliar várias dimensões de competências médicas, incluindo exame físico, obtenção de história clínica, interpretação de exames complementares, competências de comunicação, aptidões médico-cirúrgicas e atitudes.

Neste curso especial, os portfólios reflexivos constituíram um meio de acompanhamento contínuo dos processos relativos ao desenvolvimento pessoal e profissional, e foram enviados de forma digital e discutidos com o docente uma vez por semana, em reuniões de uma hora. O docente analisou a qualidade dos relatórios, detectou as deficiências existentes e respondeu ao portfólio, atuando como mentor. No momento do encontro semanal, discutiram-se os casos mais interessantes, ocorrendo a revisão do exame físico de pacientes escolhidos e exercício do raciocínio clínico e Medicina Baseada em Evidências.

$\mathrm{Na}$ avaliação de Habilidades e Atitudes por meio do Osce, incorporam-se, algumas vezes, pacientes simulados por atores, que são treinados para retratar pacientes da vida real, em repetidas estações, cronometradas e em diferentes tarefas. Neste momento, os examinados se alternam por um número determinado de estações, onde se encontram pacientes reais ou simulados, com o propósito de realizar diferentes tarefas clínicas, focadas em anamnese, exame físico e comunicação com pacientes e familiares. Habitualmente, professores avaliadores observam os examinados e registram os aspectos do desempenho com base em checklist previamente estruturado para avaliar habilidades clínicas e atitudes.

A avaliação de competências clínicas que permite analisar as condições de desenvolvimento dos progressos individuais foi realizada por meio de discussão de casos clínicos, portfólios reflexivos e desempenho no Osce. A avaliação formativa está muito ligada ao mecanismo de realimentação, já que permite ao professor detectar e identificar deficiências na forma de ensinar, possibilitando reformulações em seu trabalho didático, visando aperfeiçoá-lo quando ainda em curso ${ }^{5}$.
A avaliação educacional realizada pelo docente responsável pelas Habilidades e Atitudes está sumarizada no Quadro 2.

QUADRO 2

Avaliação educacional do Módulo de Habilidades e Atitudes

FICHA DE AVALIAÇÃO DE HABILIDADES E PORTFÓLIOS

Nome do aluno:

Avaliador: Assinatura:

Data:

\begin{tabular}{c|c|c|c|c}
$\begin{array}{c}\text { HABILIDADES } \\
\text { GERAIS }\end{array}$ & $\begin{array}{c}\text { Realizou } \\
\text { adequado }\end{array}$ & $\begin{array}{c}\text { Realizou } \\
\text { inadequado }\end{array}$ & $\begin{array}{c}\text { Não } \\
\text { realizou }\end{array}$ & $\begin{array}{c}\text { Necessário } \\
\text { retreinamento }\end{array}$ \\
\hline
\end{tabular}

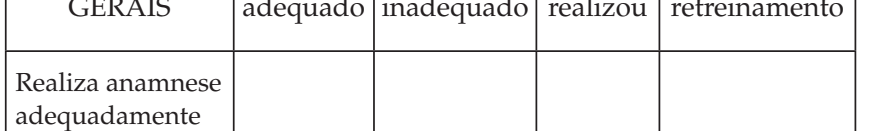

Executa

exame físico

adequadamente

Solicita exames

complementares

para o raciocínio

clínico

Formulação

diagnóstica

abrangente

Eixo I -

Transtornos

clínicos;

Eixo II -

Incapacidades;

Eixo III — Fatore

contextuais;

Eixo IV -

Qualidade de

vida.

Plano terapêutico

e preventivo

baseado em

evidências

científicas

Avaliou,

interpretou

e aplicou a

literatura médica

Associou com as

ciências básicas?

Associou com a

fisiopatogenia?

Associação

com aspectos

psicossociais

(contexto

ambiental,

emocional,

familiar) 


\begin{tabular}{|l|l|l|l|l|}
\hline $\begin{array}{l}\text { Avaliou a } \\
\text { incapacidade } \\
\text { (ocupacional, } \\
\text { familiar e social) }\end{array}$ & & & & \\
\hline $\begin{array}{l}\text { Habilidades de } \\
\text { comunicação } \\
\text { com paciente e } \\
\text { familiares }\end{array}$ & & & & \\
\hline PORTFÓLIOS & $\begin{array}{c}\text { Entregue } \\
\text { em }\end{array}$ & $\begin{array}{c}\text { Entregue } \\
\text { em }\end{array}$ & $\begin{array}{c}\text { Entregue } \\
\text { em }\end{array}$ & \\
\hline $\begin{array}{l}\text { Progressos } \\
\text { individuais na } \\
\text { discussão dos } \\
\text { casos clínicos, } \\
\text { portfólios } \\
\text { reflexivos e Osce }\end{array}$ & & & & \\
\hline
\end{tabular}

\section{CONSIDERAÇÕES FINAIS}

O ensino de Habilidades e Atitudes tem como referência o modelo biopsicossocial, e os métodos empregados para avaliar a aprendizagem das competências clínicas são baseados no exame Osce, na discussão de casos clínicos, nos portfólios reflexivos e no feedback.

A experiência aqui descrita e vivenciada pelos professores no ensino de Habilidades e Atitudes permitiu a integração entre o conhecimento dedutivo e cognitivo da prática clínica e um sistema de vários métodos de avaliação de competências clínicas. Destaca-se como fator extremamente positivo o treinamento e a capacitação docente para aprimorar o ensino de Habilidades e Atitudes no currículo integrado dentro do modelo biopsicossocial. Este modelo tem como pilar o uso de metodologias ativas, a interdisciplinaridade e a valorização da avaliação no processo de formação ${ }^{18}$.

Em síntese, julgamos necessário o apoio institucional para garantir o aperfeiçoamento contínuo do corpo docente para o ensino de Habilidades e Atitudes e uma educação permanente em formação e prática em saúde.

A experiência de um grupo de docentes da área de Clínica Médica na aquisição de competências em Habilidades e Atitudes visou aprimorar a formação e prática em saúde em um processo de ensino-aprendizagem do tipo ABP, ancorado em modelo biopsicossocial e utilizando estratégias de avaliações formativas.

\section{REFERÊNCIAS}

1. Thagard P, Litt A. (forthcoming). Models of scientific explanation. In: R. Sun (Ed.), The Cambridge handbook of computational cognitive modeling. Cambridge: Cambridge University Press; 2006.
2. Epstein R. Assessment in Medical Education. N Engl J Med. 2007; 356: 387-396.

3. Neves F, Pazin- Filho A. Raciocínio Clínico na Sala de Urgência. Medicina, Ribeirão Preto, 2008; 41 (3): 339-46.

4. Piovezan RD, Custodio O, Cendoroglo MS, Batista NA. Teste de concordância de scripts: uma proposta para a avaliação do raciocínio clínico em contextos de incerteza. Rev Bras Educ Méd. 2010; 34(1): 05-12.

5. Epstein R, Hundert E. Defining and assessing professional competence. JAMA. 2002; 287: 226-235.

6. Faria MJ, Nunes EF, Anastasiou L, Sakai M, Silva V. Os desafios da educação permanente: a experiência do curso de Medicina da Universidade Estadual de Londrina Rev Bras Educ Méd. 2008; 32(2): 248-253.

7. Lima GZ, Linhares RE. Escrever bons problemas. Rev Bras Educ Méd. 2008; 32(2): 197-201.

8. Nunes SOV, Vargas HO, Bueno C, Rocha R, Mangili L. O ensino da Psiquiatria na Graduação de Medicina pelo Método de Aprendizagem Baseada em Problemas. Psiquiatria Biológica, 2002; 10: 23-26.

9. Nunes SOV, Vargas HO, Liboni M, Martins D, Vargas LHM, Turini B. O ensino da Psiquiatria, habilidades de comunicação e atitudes no currículo integrado da Medicina da Universidade Estadual de Londrina. Rev Bras Educ Méd . 2008; 32(2): 210-216.

10. Turini B, Martins D, Tavares M, Nunes SOV, Silva V, Thomson Z. Comunicação no ensino médico: estruturação, experiência e desafios em novos currículos. Rev Bras Educ Méd. 2008; 32(2): 264-270.

11. Campos JJB, Elias PE. A saúde coletiva no curso de medicina da Universidade Estadual de Londrina: reflexões iniciais. Rev Bras Educ Méd. 2008; 32(2): 149-159.

12. Vargas LH, Colus IM, Linhares RE, Salomão TMS, Marchese MC. Inserção das ciências básicas no currículo do curso de Medicina da Universidade Estadual de Londrina. Rev Bras Educ Méd. 2008; 32(2): 174-179.

13. Lopes AA. Medicina Baseada em Evidências: a arte de aplicar o conhecimento científico na prática clínica. Rev. Assoc. Med. Bras. 2000; 46(3): 285-8.

14. Thagard P. (forthcoming). Patterns of medical discovery. In: F. Gifford (Ed.), Handbook of philosophy of medicine. Amsterdam: Elsevier; 2009.

15. Rea-Neto A. Raciocínio clínico - o processo de decisão diagnóstica e terapêutica. Rev Ass Med Brasil 1998; 44 (4): 301-11.

16. Marco M. Do modelo biomédico ao modelo biopsicossocial: um projeto de educação permanente. Rev Bras Educ Méd. 2006; 30(1): 60-72. 
17. IGDA WORKGROUP, WPA IGDA. 7: Standardized multi-axial diagnostic formulation. B J Psych. 2003; 182: s52-s54.

18. Perim G, Sakai M, Almeida M, Marchese M, Matsuo T. A avaliação Institucional no curso de Medicina da Universidade de Londrina: uma experiência inovadora. . Rev Bras Educ Méd. 2008; 32(2): 217-229.

\section{CONTRIBUIÇÃO DOS AUTORES}

Todos os autores participaram na concepção e desenho deste estudo ou da análise e interpretação dos dados, assim como da redação deste texto.
CONFLITO DE INTERESSES

Declarou não haver.

\section{ENDEREÇO PARA CORRESPONDÊNCIA}

Sandra Odebrecht Vargas Nunes

Av. Adhemar de Barros, 625

Londrina - Paraná

CEP 86050-190 — PR

E-mail: sandranunes@sercomtel.com.br 